\title{
10 AUTOEFICACIA EN CUIDADORES DE PERSONAS CON DISCAPACIDAD: REVISIÓN INTEGRATIVA
}

\author{
| Martha Parra-Aguirre' Patrícia Cid-Henríquez²; Alda Orellana-Yáñez $^{3} \mid$
}

\section{RESUMEN}

CONTEXTO: La capacidad de los cuidadores familiares para enfrentar las demandas del cuidado en el hogar se desarrolla individualmente según la capacidad de autoeficacia que logre alcanzar a lo largo de la vida.

OBJETIVO: Conocer la percepción de autoeficacia de los cuidadores familiares de personas con discapacidad.

METODOLOGÍA: Se utilizó el formato PICO para la pregunta de investigación. Se buscaron artículos científicos en las bases de datos Scopus, PubMed, SciELO, BVS y WOS, teniendo en cuenta los descriptores: autoeficacia, cuidadores y personas con discapacidad, con sus respectivas traducciones al inglés y portugués. Se establecieron cuatro estrategias de búsqueda diferentes utilizando el booleano AND. Los filtros aplicados fueron: texto completo y límite de temporalidad 2008 a 2018.

RESULTADOS: Se identificaron 74 estudios de los cuales se eliminaron 68, finalmente, el estudio incluyó 6 investigaciones que evalúan la percepción de autoeficacia en el cuidador familiar de personas con discapacidad. Los estudios reportan a la autoeficacia como un mecanismo de afrontamiento con fuerte validez predictiva en las condiciones de salud y los dominios de las conductas de salud. La asociación más comúnmente reportada es entre la autoeficacia y la sobrecarga percibida, en donde existe una asociación lineal negativa.

CONCLUSIONES: La autoeficacia es un determinante poderoso y modificable de la conducta por lo que debe ser foco de atención de los profesionales de enfermería. Surge la necesidad de definir modelos de intervención en enfermería en conjunto con las familias a través de un acompañamiento a lo largo del tiempo.

DESCRIPTORES: Autoeficacia; Cuidadores; Personas con discapacidad

\section{RESUMO}

"Auto-eficácia em cuidadores de pessoas com deficiência: Revisão integrativa"

CONTEXTO: A capacidade dos cuidadores familiares de atender às demandas do cuidado domiciliar é desenvolvida individualmente, de acordo com a capacidade de autoeficácia que eles alcançam ao longo da vida. OBJETIVO: Conhecer a percepção de autoeficácia de cuidadores familiares de pessoas com deficiência.

MÉTODOS: Foi utilizado o formato PICO para a questão de pesquisa. Pesquisámos artigos científicos nas bases de dados Scopus, PubMed, SciELO, BVS e WOS, levando em consideração os descritores: autoeficácia, cuidadores e pessoas com deficiência, com suas respectivas traduções para inglês e português. Foram estabelecidas quatro estratégias de busca diferentes usando o AND booleano. Os filtros aplicados foram: texto completo e prazo de 2008 a 2018.

RESULTADOS: Foram identificados 74 estudos, dos quais 68 foram eliminados e, por fim, foram incluídos 6 estudos que avaliam a percepção de autoeficácia no cuidador familiar de pessoas com deficiência. Estudos relatam a autoeficácia como um mecanismo de enfrentamento com forte validade preditiva nas condições de saúde e nos domínios dos comportamentos de saúde. A associação mais comumente relatada é entre autoeficácia e sobrecarga percebida, onde há uma associação linear negativa. CONCLUSÕES: A autoeficácia é um determinante poderoso e modificável do comportamento, devendo ser o foco dos profissionais de enfermagem. Surge a necessidade de definir modelos de intervenção em enfermagem junto às famílias por meio de um acompanhamento ao longo do tempo.

\section{PALAVRAS-CHAVE: Autoeficácia; Cuidadores; Pessoas com deficiência}

\begin{abstract}
"Self-efficacy in caregivers of persons with disabilities: Integrative review"

BACKGROUND: The capacity of family caregivers to meet the demands of home care is developed individually according to the capacity of selfefficacy that they achieve throughout life.

AIM: To know the perception of self-efficacy of family caregivers of people with disabilities.

METHODS: The PICO format was used for the research question. Scientific articles were searched in the Scopus, PubMed, SciELO, BVS and WOS databases, taking into account the descriptors: self-efficacy, caregivers and people with disabilities, with their respective translations into English and Portuguese. Four different search strategies were established using the AND boolean. The filters applied were: full text and time limit from 2008 to 2018.

RESULTS: 74 studies were identified, 68 of which were eliminated. Finally, the study included 6 studies that evaluate the perception of self-efficacy in the family caregiver of people with disabilities. Studies report self-efficacy as a coping mechanism with strong predictive validity in health conditions and domains of health behaviors. The most commonly reported association is between self-efficacy and perceived overload, where there is a negative linear association.

CONCLUSIONS: Self-efficacy is a powerful and modifiable determinant of behavior, so it should be the focus of nursing professionals. The need arises to define models of intervention in nursing together with families through an accompaniment over time.
\end{abstract}

\section{KEYWORDS: Self-efficacy; Caregivers; Disabled persons}

Submetido em 30-09-2019

Aceite em 21-01-2020

\footnotetext{
1 Doutoranda em Enfermagem na Universidad de Concepción, Chile; Docente de Enfermagem na Universidad Central del Ecuador, Quito, Equador, maparraa@uce.edu.ec 2 Doutora em Enfermagem; Professora Titular na Universidad de Concepción, Concepción, Chile, patcid@udec.cl

3 Doutora em Enfermagem; Professora Associada na Universidad de Concepción, Casilla, Concepción, Chile, aorellan@udec.cl
} 


\section{INTRODUCCIÓN}

El incremento de la esperanza de vida al nacer y la progresión constante de las enfermedades crónicas repercuten en el desarrollo de discapacidades y condiciones de dependencia, lo cual también implica un aumento en la necesidad de personas que se dediquen a brindar cuidados en el ambiente familiar.

El cuidado de un paciente dependiente supone un exceso de trabajo para los cuidadores a cargo, con largas jornadas y altas horas de constante dedicación (Pinzón \& Carrillo, 2016). Esta circunstancia genera ansiedad, agotamiento y temor de no cumplir de forma adecuada con las labores de asistencia, lo cual puede repercutir en sentimientos de culpa que más adelante se ven reflejados en sobrecarga y alteración en la calidad de vida (Pinzón \& Carrillo, 2016). Sin embargo, aun presentando algunos problemas en la experiencia de asistencia, a menudo los cuidadores son capaces de mostrar una extraordinaria capacidad de adaptación a las circunstancias que les ha tocado vivir (Crespo \& FernándezLansac, 2015).

Las personas pueden afrontar las presiones como amenaza o como oportunidad en las diferentes etapas del curso de la vida, ejerciendo su papel activo y concentrándose en las posibilidades que las situaciones proporcionan, en función de sus creencias sobre su capacidad de actuar sobre ellas (Fontes \& Azzi, 2012). En esta transición potencialmente estresante, como es hacia el papel de cuidador, las creencias de autoeficacia, pueden servir como recurso personal facilitador o factor de vulnerabilidad (Petronilho, Pereira \& Silva, 2015).

Para explicar estos procesos de afrontamiento, surgen desde la psicología modelos que han puesto de relieve el papel de la cognición en la regulación del comportamiento y dentro de ellos se destaca el concepto de autoeficacia, el cual en el ámbito de la salud cobra importancia en la prevención de conductas riesgosas y en la promoción de comportamientos que van en beneficio de las personas (Zenteno, Cid \& Saez, 2017).

La autoeficacia es el constructo más importante dentro de la teoría social-cognitiva propuesta por Bandura (1977), donde se enfatiza el papel de los fenómenos autorrefenciales como el medio por el cual el ser humano es capaz de actuar en su ambiente y transformarlo, permitiendo a las personas crear y desarrollar autopercepciones acerca de sus capacidades y estas se conviertan en los medios por los cuales siguen sus metas y toman sus decisiones (Ornelas, Blanco, Aguirre \& Guedea, 2012).
Las creencias de autoeficacia determinan el nivel de motivación, la cantidad de esfuerzo y tiempo empleado para alcanzar un objetivo (Pereira \& Almeida, 2004). Esta creencia acerca de lo que es capaz de realizar, da forma a las acciones, cuanto más fuerte la creencia en las capacidades personales, mayor y más largos serán los esfuerzos. Así, las creencias de autoeficacia pueden transformar cognitivamente las situaciones que parecen ser amenazantes en situaciones manejables (Ornelas et al., 2012; Pereira \& Almeida, 2004; Taylor \& IrizarryRobles, 2015).

La percepción de autoeficacia se enmarca en el control personal de las acciones, no refleja las competencias personales, sino las creencias, juicios y evaluaciones sobre lo que se puede realizar con las competencias que se posee en situaciones determinadas y tiene una elevada predictibilidad en relación con las tareas de comportamiento específico (Pereira \& Almeida, 2004). Estas creencias no son uniformes, y provienen de: experiencias vividas; persuasión verbal de otros; modelación social por medio de la observación del desempeño de otras personas; y el estado físico y emocional durante una tarea, como un reflejo de las capacidades y limitaciones personales para adaptarse al dominio particular de funcionamiento que es el objeto de interés (Bandura, 2006; Pace et al., 2017).

En este caso se trata de la autoeficacia del cuidador, la cual puede explicar la variabilidad en la capacidad de los miembros de la familia para hacer frente a las demandas crónicas de los cuidados y evaluar la confianza de los cuidadores para responder a estos desafíos según las tareas a desarrollar (Steffen, McKibbin, Zeiss, Gallagher-Thompson \& Bandura, 2002). De este modo, la percepción de autoeficacia, le permitirá al cuidador organizar y ejecutar un cuidado que sea eficiente y que permita prevenir conductas mal adaptativas o riesgosas entre la diada cuidado y cuidador (Zenteno et al., 2017). Dentro del contexto del cuidado familiar ha existido un interés permanente por comprender los factores cognitivos y comportamentales que favorecen o dificultan el ejercicio del rol de cuidador. Esto ha llevado a evaluar la autoeficacia del cuidado, identificándose tres dimensiones: autocuidado y obtención de respiro, manejo de conductas disruptivas y control de pensamientos perturbadores, que reflejan desafíos conductuales y cognitivos distintivos y comunes para los cuidadores familiares de personas dependientes (Steffen et al., 2018). 
Dada la importancia de la percepción de autoeficacia del cuidador para la entrega de su cuidado es que esta revisión integrativa tiene como objetivo conocer la percepción de autoeficacia de los cuidadores familiares de personas con discapacidad.

\section{METODOLOGÍA}

En el contexto actual la enfermería basada en la evidencia (EBE) constituye una pieza clave para garantizar que la práctica sea efectiva y eficiente utilizando en forma crítica los resultados de la investigación científica para establecer mejoras en la calidad de los cuidados de enfermería (Subirana \& Fargues, 2004), dando el apoyo científico necesario para buscar y evaluar la evidencia y posteriormente en conjunto con la experiencia clínica tomar las decisiones en el cuidado (Orellana \& Paravic, 2007).

En esta investigación se utilizó la metodología de la revisión integrativa sugerida por Mendes, Silveira \& Galvão (2008), cuyas etapas son: definición del problema y formulación de la pregunta de investigación, establecimiento de criterios para inclusión y exclusión de estudios, búsqueda en las bases de datos para identificación de los estudios a ser incluidos en la revisión, definición de la información a extraer de los estudios seleccionados y categorización de los estudios, evaluación de los estudios incluidos en la revisión integrativa, interpretación de los resultados y finalmente, síntesis del conocimiento.

Para la formulación de la pregunta de investigación se utilizó el formato PICO, incluyendo tres de sus componentes: ¿Cuál es la percepción de autoeficacia de los cuidadores familiares de personas con discapacidad?

Para la elección de los estudios se aplicaron los siguientes criterios de inclusión: artículos originales, disponibles en texto completo, en idiomas inglés, español o portugués, correspondientes a los años 2008 a julio 2018 y que contuvieran de manifiesto en el texto algún tipo de relación entre autoeficacia y cuidador de personas con discapacidad. Se excluyeron estudios de intervención y estudios para validación de escalas.

Se realizó la búsqueda de los artículos científicos en las bases de datos Scopus, PubMed, SciELO, BVS y WOS. La búsqueda se orientó tomando en cuenta los descriptores MeSH/DeCS: cuidadores, personas con discapacidad, autoeficacia y sus respectivas traducciones al idioma inglés y portugués. Se establecieron cuatro estrategias de búsqueda diferentes utilizando el booleano AND según se muestra en el Cuadro 1.
Cuadro 1 - Estrategias de Búsqueda para la Selección de Estudios

\begin{tabular}{|l|l|l|}
\hline Base de datos & Estrategia de búsqueda & $\begin{array}{l}\text { Artículos } \\
\text { encontrados }\end{array}$ \\
\hline \multirow{2}{*}{ BVS } & $\begin{array}{l}\text { Caregivers and Disabled Per- } \\
\text { sons and Self Efficacy }\end{array}$ & 17 \\
\hline \multirow{5}{*}{ PubMed } & $\begin{array}{l}\text { Caregivers and Disabled Per- } \\
\text { sons and Self Efficacy }\end{array}$ & 8 \\
\hline \multirow{3}{*}{ Scopus } & $\begin{array}{l}\text { Caregivers and Disabled Per- } \\
\text { sons and Self Efficacy }\end{array}$ & 2 \\
\cline { 2 - 3 } & $\begin{array}{l}\text { Family Caregiver and person } \\
\text { with Physical Disabilities and } \\
\text { Self Efficacy }\end{array}$ & 3 \\
\cline { 2 - 4 } & $\begin{array}{l}\text { Family Caregiver and person } \\
\text { with Physical Disabilities }\end{array}$ & 16 \\
\hline \multirow{2}{*}{ SciELO } & $\begin{array}{l}\text { Caregivers and Disabled Per- } \\
\text { sons and Self Efficacy }\end{array}$ & 13 \\
\hline & $\begin{array}{l}\text { Caregivers and Disabled Per- } \\
\text { sons and Self Efficacy }\end{array}$ & 0 \\
\cline { 2 - 4 } & Cuidadores e Auto Eficácia & 15 \\
\hline
\end{tabular}

El procedimiento para la selección de artículos se resume en la Figura 1.

Figura 1 - Diagrama de flujo del proceso de selección de estudios para revisión integrativa

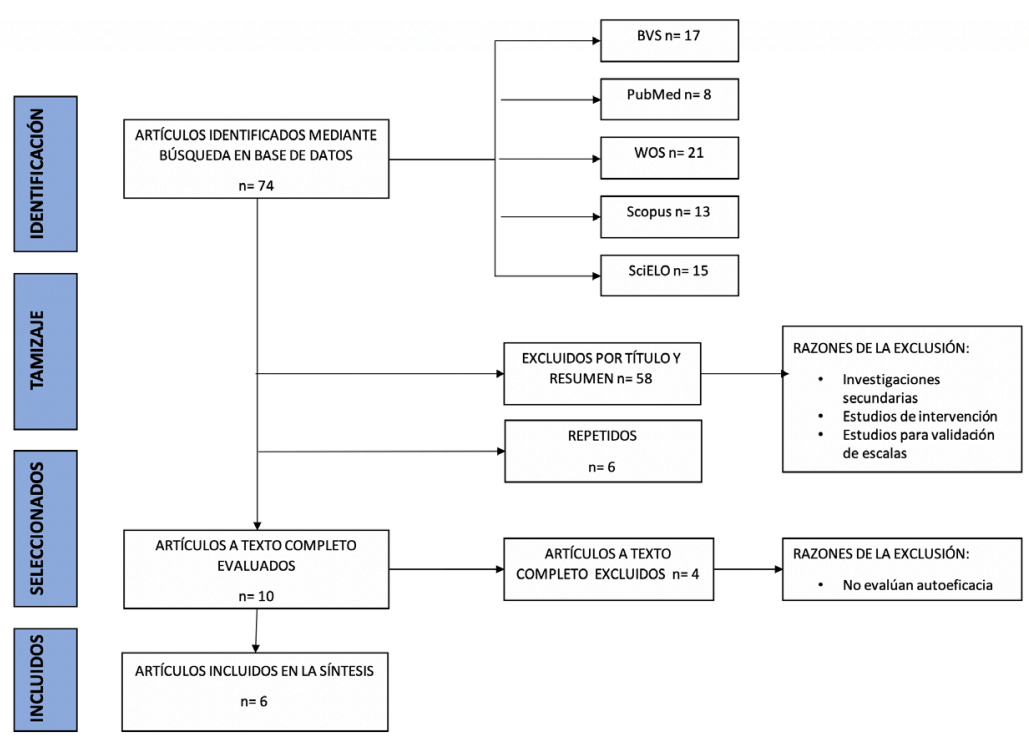

Los artículos identificados en las bases de datos se exportaron al gestor de referencias Zotero para la eliminación de los duplicados y la aplicación de los criterios de inclusión y exclusión. Finalizada esta etapa, se utilizó un instrumento (ficha resumen) para la extracción, categorización y evaluación de la información de los artículos seleccionados, incluyendo autor, título, revista, país, año de publicación, lugar de estudio, objetivo, metodología, resultados y conclusiones relevantes. Para garantizar la calidad de la evidencia seleccionada se realizó el análisis crítico de los artículos científicos y se aplicó las tres preguntas claves para estudios cuantitativos: 
¿Son válidos los resultados?; ¿Cuáles son los resultados?; ¿Resultan útiles los resultados? (Subirana \& Fargues, 2004), con lo cual se eliminaron en la lectura a texto completo cuatro artículos.

\section{RESULTADOS}

De los seis artículos el 100\% de los estudios corresponden al enfoque cuantitativo, cinco de las investigaciones son de corte transversal y solamente la investigación de Petronilho et al. (2015) es de tipo longitudinal. Los análisis estadísticos aplicados son de tipo descriptivo y correlacional.

Respecto al año de publicación, dos corresponden al 2013, dos al 2015 y solamente uno para los años 2016 y 2017. La mitad de las investigaciones fueron publicadas en revistas de enfermería y la otra mitad en revistas de psicología y medicina de la familia.

En cuanto al lugar de la investigación, cuatro de las seis publicaciones corresponden a países de la Unión Europea y solamente dos corresponden a América Latina. Los artículos que compusieron el corpus de análisis se presentan en el Cuadro 2 ordenados según el año de publicación.

Cuadro 2 - Descripción de las Publicaciones sobre Autoeficacia en el Cuidador de Personas con Discapacidad

\begin{tabular}{|c|c|c|c|}
\hline $\begin{array}{l}\text { Autor } \\
\text { País identificado }\end{array}$ & Título & $\begin{array}{l}\text { Revista } \\
\text { Año }\end{array}$ & Aporte a la comprensión del fenómeno estudiado \\
\hline $\begin{array}{l}\text { Marrón, Redolar- } \\
\text { Ripol, Boixadós, Nieto, } \\
\text { Guillamón, Hernández } \\
\text { \& Gómez. } \\
\text { España. }\end{array}$ & $\begin{array}{l}\text { Burden on Caregivers of } \\
\text { Children with Cerebral } \\
\text { Palsy: Predictors and } \\
\text { Related Factors }\end{array}$ & $\begin{array}{l}\text { Universitas } \\
\text { Psychologica } \\
2013\end{array}$ & $\begin{array}{l}\text { La autoeficacia tenía una asociación lineal negativa con la carga, mien- } \\
\text { tras que el grado de discapacidad y depresión una asociación lineal } \\
\text { positiva. Los predictores más importantes de la carga de los cuida- } \\
\text { dores fueron el grado de discapacidad, la depresión y la autoeficacia. }\end{array}$ \\
\hline $\begin{array}{l}\text { Guedes \& Pereira. } \\
\text { Portugal. }\end{array}$ & $\begin{array}{l}\text { Burden, Coping, Physi- } \\
\text { cal Symptoms and Psy- } \\
\text { chological Morbidity in } \\
\text { Caregivers of Function- } \\
\text { ally Dependent Family } \\
\text { Members }\end{array}$ & $\begin{array}{l}\text { Revista } \\
\text { Latino- } \\
\text { Americana. } \\
\text { Enfermagem } \\
2013\end{array}$ & $\begin{array}{l}\text { Fue encontrada una correlación negativa significativa entre estrategias } \\
\text { de enfrentamiento entre ellas la autoeficacia y las diferentes variables } \\
\text { clínicas del cuidador, y también una correlación positiva significativa } \\
\text { entre estrategias de enfrentamiento y la duración del cuidado. Los cui- } \\
\text { dadores de familiares con déficit cognitivo relatan mayor sobrecarga } \\
\text { en el relacionamiento interpersonal y menor auto-eficacia percibida. }\end{array}$ \\
\hline $\begin{array}{l}\text { Petronilho, Pereira \& } \\
\text { Silva. } \\
\text { Portugal. }\end{array}$ & $\begin{array}{l}\text { Perceção de autoeficácia } \\
\text { do familiar cuidador } \\
\text { após o regresso a casa } \\
\text { do dependente: Estudo } \\
\text { longitudinal }\end{array}$ & $\begin{array}{l}\text { Revista Por- } \\
\text { tuguesa de } \\
\text { Enfermagem } \\
\text { de Saúde } \\
\text { Mental } \\
2015\end{array}$ & $\begin{array}{l}\text { Se verificó una evolución positiva en la percepción de autoeficacia de } \\
\text { los cuidadores familiares a lo largo de tres meses tras el regreso a casa } \\
\text { del dependiente. Existe una construcción mental del papel a ejercer } \\
\text { que determina su acción, la mayor asignación de importancia a deter- } \\
\text { minados cuidados está asociada a una mayor percepción de autoefica- } \\
\text { cia. La mayor percepción de autoeficacia en la ayuda de los cuidadores } \\
\text { fue al "andar", "vestirse/desvestirse", "alimentarse" y "bañarse". }\end{array}$ \\
\hline $\begin{array}{l}\text { Crespo \& Fernández- } \\
\text { Lansac. } \\
\text { España. }\end{array}$ & $\begin{array}{l}\text { Resiliencia en cui- } \\
\text { dadores familiares } \\
\text { de personas mayores } \\
\text { dependientes }\end{array}$ & $\begin{array}{l}\text { Anales de } \\
\text { Psicología } \\
2015\end{array}$ & $\begin{array}{l}\text { Se analizaron las diferencias entre dos grupos: alta vs. moderada o baja } \\
\text { resiliencia. Aunque hubo diferencias en el estado cognitivo del recep- } \\
\text { tor de cuidado, los cuidadores con alta resiliencia mostraron mayores } \\
\text { puntuaciones significativas estadísticamente en: satisfacción con el } \\
\text { apoyo social recibido, autoeficacia para controlar los pensamientos } \\
\text { negativos, autoestima, autocuidado y extraversión. }\end{array}$ \\
\hline $\begin{array}{l}\text { Pablo, Domínguez, } \\
\text { Peláez, Rincón \& Orea. } \\
\text { México. }\end{array}$ & $\begin{array}{l}\text { Sobrecarga y autoefi- } \\
\text { cacia percibida en } \\
\text { cuidadores primarios } \\
\text { informales de pacien- } \\
\text { tes con insuficiencia } \\
\text { cardíaca }\end{array}$ & $\begin{array}{l}\text { Revista } \\
\text { Clínica de } \\
\text { Medicina de } \\
\text { Familia } \\
2016\end{array}$ & $\begin{array}{l}\text { Se observó una correlación significativa negativa entre la sobrecarga } \\
\text { percibida y autoeficacia percibida, moderada e inversamente propor- } \\
\text { cional. El 49,5 \% de la muestra reportó un nivel de autoeficacia bajo. El } \\
27,5 \% \text { presentó sobrecarga leve y el } 25,7 \% \text { sobrecarga intensa. }\end{array}$ \\
\hline $\begin{array}{l}\text { Zenteno, Cid \& Sáez. } \\
\text { Chile. }\end{array}$ & $\begin{array}{l}\text { Autoeficacia del cuida- } \\
\text { dor familiar de la per- } \\
\text { sona en estado crítico }\end{array}$ & $\begin{array}{l}\text { Enfermería } \\
\text { Universitaria } \\
2017\end{array}$ & $\begin{array}{l}\text { Se observó cómo la percepción de autoeficacia se ve influenciada } \\
\text { por las características biopsicosociales del cuidador (edad, nivel de } \\
\text { estudios, autoestima, conocimiento del tratamiento) e indicadores } \\
\text { de morbilidad de la persona cuidada en estado crítico y cómo esta se } \\
\text { relaciona con la decisión de adoptar una conducta promotora de salud } \\
\text { frente a su autocuidado y el de su familiar. }\end{array}$ \\
\hline
\end{tabular}


En cuanto a las características de los cuidadores, los estudios revelan que el perfil del cuidador se mantiene como se ha conocido tradicionalmente, es decir, mayoritariamente femenino (entre el 73\% al 88\%) (Crespo \& Fernández-Lansac, 2015; Marrón et al., 2013; Pablo, Domínguez, Peláez, Rincón \& Orea, 2016; Zenteno et al., 2017), la edad promedio fue de 40 a 62 años (Crespo \& Fernández-Lansac, 2015; Guedes \& Pereira, 2013; Marrón et al., 2013; Pablo et al., 2016; Zenteno et al., 2017), entre el $62 \%$ al $79,8 \%$ de los cuidadores reportaron tener pareja o ser casados (Guedes \& Pereira, 2013; Pablo et al., 2016; Zenteno et al., 2017). El tiempo de cuidado promedio reportado fue entre tres y ocho años (Guedes \& Pereira, 2013; Zenteno et al., 2017), en relación al nivel de estudios, los cuidadores poseen estudios básicos y/o medios (Crespo \& Fernández-Lansac, 2015; Guedes \& Pereira, 2013; Pablo et al., 2016; Zenteno et al., 2017). Sobre la relación de parentesco, la mayoría de los estudios reportan a las madres, esposas o hijas/os de las personas dependientes como sus cuidadores (Crespo \& Fernández-Lansac, 2015; Marrón et al., 2013; Pablo et al., 2016; Zenteno et al., 2017).

El mayor aporte de estos estudios se centra en la descripción de características de los cuidadores familiares que muestran prioridad para ser intervenidas, ejemplo de ello son la autoeficacia, la sobrecarga, la depresión, la ansiedad y el apoyo social (Crespo \& Fernández-Lansac, 2015; Guedes \& Pereira, 2013; Marrón et al., 2013; Pablo et al., 2016; Petronilho et al., 2015; Zenteno et al., 2017). Las intervenciones sugeridas como producto de los estudios son en su mayoría la implementación de programas de educación y apoyo efectivo a los cuidadores familiares a través del uso de herramientas como psicoeducación y entrenamiento en solución de problemas para reducir la depresión y mejorar la autoeficacia, incorporando aspectos positivos de la adaptación a los modelos teóricos de cuidado, los cuales tradicionalmente se han venido centrando en el desarrollo de sintomatología (Crespo \& Fernández-Lansac, 2015; Guedes \& Pereira, 2013; Marrón et al., 2013; Pablo et al., 2016; Petronilho et al., 2015)

\section{DISCUSIÓN}

La preocupación de enfermería por los cuidadores familiares de personas con discapacidad es importante, considerando el aumento de la esperanza de vida al nacer, las enfermedades crónicas no transmisibles y su relación con los niveles de discapacidad y dependencia que requieren de esta figura dentro del ámbito familiar.
El constructo de la autoeficacia ha sido útil para avanzar en la investigación y la práctica psicosocial con cuidadores familiares debido a que las creencias de autoeficacia muestran una fuerte validez predictiva en las condiciones de salud y los dominios de las conductas de salud (Steffen et al., 2018), como queda demostrado en los resultados de esta revisión integrativa.

Las variables psicológicas estudiadas en los cuidadores familiares fueron: autoeficacia, resiliencia, calidad de vida, percepción de apoyo social, sobrecarga, y depresión (Crespo \& Fernández-Lansac, 2015; Guedes \& Pereira, 2013; Marrón et al., 2013; Pablo et al., 2016; Petronilho et al., 2015; Zenteno et al., 2017). De los cuales la autoeficacia resalta como uno de los predictores más importantes en la carga del cuidado.

Una de las reacciones más comúnmente reportadas como consecuencia del ejercicio del rol de cuidador es la sobrecarga percibida (Guedes \& Pereira, 2013; Marrón et al., 2013; Pablo et al., 2016), entendida como el nivel en el que el cuidar influye sobre la vida social, personal, económica y aspectos de su salud (Pablo et al., 2016). Esto se corrobora con el estudio de Pablo et al. (2016), quienes indican que la sobrecarga es una de las consecuencias negativas más reportadas relacionada con la autoeficacia percibida. La autoeficacia se asocia de forma lineal negativa con la carga (Marrón et al., 2013) y esta carga aumenta en los cuidadores de familiares con déficit cognitivo (Guedes \& Pereira, 2013).

Además, se ha investigado el papel de la autoeficacia en la regulación del estrés, la ansiedad y depresión. Así, Marrón et al. (2013), encontraron una asociación lineal positiva entre el grado de discapacidad de la persona cuidada y la depresión del cuidador con la sobrecarga en donde la autoeficacia fue el mecanismo regulador de estas percepciones. En cuanto a las estrategias de enfrentamiento, Crespo \& Fernández-Lansac (2015), reportan que los aspectos subjetivos relacionados con los recursos internos y la manera en que los cuidadores valoran la situación, son los que realmente determinan su adaptación a las circunstancias. Así en su estudio, relacionaron los factores de estrés del cuidador entre dos grupos con alta vs. moderada o baja resiliencia. Los cuidadores altamente resilientes tendieron a manifestar una menor preocupación por los problemas del paciente, y presentaron menores niveles de sintomatología depresiva y ansiosa. Además, se observaron mayores puntuaciones en: satisfacción con el apoyo social recibido, autoeficacia para controlar los pensamientos negativos, autoestima, autocuidado y extraversión (Crespo \& Fernández-Lansac, 2015). 
La construcción mental que realice el cuidador sobre la importancia de su rol determina su acción y al parecer asigna mayor importancia a determinados cuidados lo cual se asocia a una mayor percepción de autoeficacia (Petronilho et al., 2015). Otro estudio reporta una correlación positiva significativa entre estrategias de enfrentamiento y la duración del cuidado (Guedes \& Pereira, 2013), lo que indica que a medida que el cuidador se adapta a su rol, disminuye la ansiedad y se establecen estrategias de enfrentamiento y entre ellas la autoeficacia (Marrón et al., 2013; Petronilho et al., 2015). Con ello surge la necesidad de definir modelos de intervención en enfermería con mayor efectividad junto a las familias a través de un acompañamiento a lo largo del tiempo más sistemático y útil, teniendo en cuenta el significado personal atribuido al cuidado, sus expectativas, sus necesidades y los recursos disponibles (Petronilho et al., 2015).

En la práctica de enfermería, la autoeficacia es un determinante poderoso y modificable de la conducta por lo que debe ser foco de atención de los profesionales de enfermería (Weng, Dai, Wang, Huang \& Chiang, 2008), se debe abogar por el acceso y la utilización de los recursos necesarios para los cuidadores familiares como una intervención crucial desde la enfermería (Petronilho et al., 2015). La importancia de que los profesionales de enfermería conozcan cómo las personas organizan sus pensamientos y acciones radica en la capacidad de fomentar una prevención efectiva y además ser capaces de identificar las necesidades de cada situación específica, a las que tendrán que dar una respuesta eficaz en la asunción por parte de los familiares del papel de cuidadores (Petronilho et al., 2015).

\section{CONCLUSIÓN}

Esta revisión integrativa de la percepción de autoeficacia en el cuidador familiar de personas con discapacidad, la evidencia como una estrategia de afrontamiento que protege al cuidador familiar de los efectos del estrés, la ansiedad y sobrecarga prolongados al que es sometido debido a su rol.

Es importante considerar a los cuidadores como un usuario más de las intervenciones del sistema de salud y especialmente de enfermería, generando propuestas que visibilicen, evalúen y actúen en la práctica con la entrega de cuidados que promocionen su autoeficacia. Se puede percibir la necesidad emergente de más estudios en enfermería sobre intervenciones enfocadas al desarrollo de autoeficacia en este grupo poblacional.

\section{IMPLICACIONES PARA LA PRÁCTICA CLÍNICA}

Los resultados obtenidos en esta investigación destacan que la naturaleza y el alcance de la autoeficacia cambian a lo largo de la vida, cobrando aún mayor importancia cuando se asumen nuevos roles, como es el caso del cuidador familiar. Frente a esto, es necesario fomentar e implementar acciones multidisciplinarias encaminadas a apoyar a los cuidadores a fortalecer su autoeficacia para enfrentar los desafíos que le impone el ejercicio de este rol, así como para complementar los modelos de cuidado hasta ahora existentes.

\section{REFERENCIAS BIBLIOGRÁFICAS}

Bandura, A. (1977). Self-efficacy: Toward a unifying theory of behavioral change. Psychological Review, 84(2), 191-215. Doi: 10.1016/0146-6402(78)90002-4

Bandura, A. (2006). Guide for constructing Self-Efficacy Scales. In Bandura, A. Self-Efficacy beliefs of adolescents. 5(1), 307-337. Disponível em: https://www.uky. edu/ eushe2/Bandura/BanduraGuide2006.pdf

Crespo, M. \& Fernández-Lansac, V. (2015). Resiliencia en cuidadores familiares de personas mayores dependientes. Anales de Psicología, 31(1), 19-27. Disponível em: http://scielo.isciii.es/scielo.php?script=sci_ abstract\&pid=S0212-97282015000100003\&lng=es\&nr $\mathrm{m}=\mathrm{iso} \& \operatorname{tlng}=\mathrm{es}$

Fontes, A. \& Azzi, R. (2012). Self-efficacy beliefs and resilience: Findings of social-cognitive literature. Estudos de Psicologia (Campinas), 29(1), 105-114. Disponível em: http://www.scielo.br/scielo.php?script=sci_ abstract\&pid $=$ S0103-166X2012000100012\&lng=en\&n $\mathrm{rm}=\mathrm{iso} \& \ln \mathrm{g}=\mathrm{pt}$

Guedes, A. C. \& Pereira, M. D.G. (2013). Burden, coping, physical symptoms and psychological morbidity in caregivers of functionally dependent family members. Revista latino-americana de enfermagem, 21(4), 935-940. Disponível em: http://www. scielo.br/scielo.php? script=sci_abstract $\&$ pid $=$ S0104$11692013000400935 \& \operatorname{lng}=$ en $\& n r m=$ iso \&tlng=es

Marrón, E. M., Redolar-Ripol, D., Boixadós, M., Nieto, R., Guillamón, N., Hernández, E. \& Gómez, B. (2013). Burden on caregivers of children with cerebral palsy: predictors and related factors. Universitas Psychologica, 12(3), 767-777. 
Mendes, K. D. S., Silveira, R. C. de C. P. \& Galvão, C. M. (2008). Integrative literature review: a research method to incorporate evidence in health care and nursing. Texto \& Contexto-Enfermagem, 17(4), 758-764. Disponível em: http://www.scielo.br/scielo.php?script=sci_ abstract\&pid=S0104-07072008000400018\&lng=en\&nr $\mathrm{m}=\mathrm{iso} \& \operatorname{tng}=\mathrm{pt}$

Orellana, A. \& Paravic, T. (2007). Enfermería basada en evidencia: barreras y estrategias para su implementación. Ciencia y enfermería, 13(1), 17-24. Disponível em: https://scielo.conicyt.cl/scielo.php?script=sci_ abstract\&pid=S0717-95532007000100003\&lng=es\&nr $\mathrm{m}=\mathrm{iso} \& \operatorname{tlng}=\mathrm{es}$

Ornelas, M., Blanco, H., Aguirre, F. \& Guedea, J. C. (2012). Autoeficacia percibida en conductas de cuidado de la salud en universitarios de primer ingreso: Un estudio con alumnos de educación física. Perfiles educativos, 34(138), 62-74. Disponível em: http://www.scielo.org. $\mathrm{mx} /$ scielo.php? script $=$ sci_ abstract\&pid=S0185-26982012000400005\&lng=es\&nr $\mathrm{m}=\mathrm{iso} \& \operatorname{tlng}=\mathrm{es}$

Pablo, R., Domínguez, B., Peláez, V., Rincón, S. \& Orea, A. (2016). Sobrecarga y autoeficacia percibida en cuidadores primarios informales de pacientes con insuficiencia cardíaca. Revista Clínica de Medicina de Familia, 9(3), 152-158. Disponível em: http://scielo.isciii.es/scielo.php? script $=$ sci_abstract\&pid=S1699-695X2016000 $300004 \& \operatorname{lng}=\mathrm{pt} \& \mathrm{nrm}=\mathrm{iso} \& \ln \mathrm{l}=\mathrm{es}$

Pace, A. E., Gomes, L. C., Bertolin, D. C., Loureiro, H. M. A. M., Bijl, J. V. D. \& Shortridge-Baggett, L. M. (2017). Adaptation and validation of the Diabetes Management Self-Efficacy Scale to Brazilian Portuguese. Revista latino-americana de enfermagem, 25.

Pereira, M. G. \& Almeida, P. (2004). Auto-eficácia na diabetes: Conceito e validação da escala. Análise Psicológica, 22(3), 585-595. Disponível em: http://publicacoes.ispa.pt/index.php/ap/article/view/228

Petronilho, F. A. S., Pereira, F. M. S. \& Silva, A. A. D. (2015). Perceção de autoeficácia do familiar cuidador após o regresso a casa do dependente: Estudo longitudinal. Revista Portuguesa de Enfermagem de Saúde Mental, (SPE2), 09-13. Disponível em: http://www.scielo.mec.pt/scielo.php?script=sci_abstract\&pid=S1647$21602015000100002 \& \operatorname{lng}=\mathrm{pt} \& \mathrm{nrm}=\mathrm{iso} \& \operatorname{tng}=\mathrm{pt}$
Pinzón, E. A. \& Carrillo, G. M. (2016). Carga del cuidado y calidad de vida en cuidadores familiares de personas con enfermedad respiratoria crónica. Revista Facultad Nacional de Salud Pública, 34(2), 193-201. Disponível em: http://www.scielo.org.co/pdf/rfnsp/ v34n2/v34n2a08.pdf

Steffen, A. M., McKibbin, C., Zeiss, A. M., GallagherThompson, D. \& Bandura, A. (2002). The revised scale for caregiving self-efficacy: Reliability and validity studies. The Journals of Gerontology Series B: Psychological sciences and social sciences, 57(1), 74-86. Disponível em: https://academic.oup.com/psychsocgerontology/ article/57/1/P74/576212

Steffen, A., Gallagher-Thompson, D., Arenella, K., Au, A., Cheng, S., Crespo, M., Cristancho-Lacroix, V., López, J., Losada-Baltar, A., Márquez-González, M., NogalesGonzález, C. \& Romero-Moreno, R. (2018). Validating the revised scale for caregiving self-efficacy: a crossnational review. The Gerontologist, 59(4), e325-e342. Disponível em: https://academic.oup.com/gerontologist/article-abstract/59/4/e325/4930706?redirectedFro $\mathrm{m}=$ fulltext

Subirana, M. \& Fargues, I. (2004). Enfermería Basada en la Evidencia. En Alonso, P., Esquerro, O., Fargues, I., Garcia, J., Marzo, M., Navarra, M., Pardo, J. \& Urrutia, G. Enfermería Basada en la Evidencia. Hacia la excelencia en los cuidados. Material didáctico de apoyo para profesionales de enfermería (pp.10-24.). Madrid: Difusión Avances de Enfermería.

Taylor, J. A. S. \& Irizarry-Robles, C. Y. (2015). Factores protectores de la depresión en una muestra de adultos mayores en Puerto Rico: autoeficacia, escolaridad y otras variables socio-demográficas. Acta Colombiana de Psicología, 18(1), 125-134. Disponível em: https:// www.redalyc.org/articulo.oa?id=79838614012

Weng, L.C., Dai, Y. T., Wang, Y.W., Huang, H.L. \& Chiang, Y. J. (2008). Effects of self-efficacy, self-care behaviours on depressive symptom of Taiwanese kidney transplant recipients. Journal of Clinical Nursing, 17(13), 17861794. Disponível em: https://onlinelibrary.wiley.com/ doi/abs/10.1111/j.1365-2702.2007.02035.x

Zenteno, A., Cid, P. \& Saez, K. (2017). Autoeficacia del cuidador familiar de la persona en estado crítico. Enfermería Universitaria, 14(3), 146-154. 